G. SWAR UP and C. R. SUBRAHMANYA

Radio Astronomy Center, Tata Institute of Fundamental

Res ea rch, P. O. Box 8, Oota camund, 643001, India

\title{
SUMMAR Y
}

The median values of angular sizes of weak extragalactic radio sources, the flux densities of which lie in the range of about 0.3 to $5 \mathrm{Jy}$ at $327 \mathrm{MHz}$, have been determined for a new sample of 119 sour ces observed during 1973-74, and agree well with the value of about 10 arc sec determined earlier by Swarup (1975). For 8 different flux density ranges, the angular size distribution for the All-sky, 3CR and Ooty radio sources have been compared with theoretical predictions based on the evolutionary model by Kapahi (1975) and show a remarkable agreement with his model except that the best fit is found for a linear size evolution proportional to $(1+z)^{-1}$.

\section{INTRODUCTION}

In this paper are presented some new data on the flux densityangular size distribution, $\mathrm{N}(\mathrm{S}, \theta)$, for extragalactic radio sources and these data are used for cosmological investigations. Recently, Kapahi (1975) presented an independent evidence of evolution in source properties with cosmological epoch by examining simultaneously (a) the angular size distribution $N(>\theta)$ for the $3 C R$ radio sources and (b) the relation between the median value of maximum angular extent, $\theta_{\mathrm{m}}$, and flux density $S$ derived by Swarup (1975). Here we have examined all the available data, i. e. angular size distributions $N(S, \theta)$ for 8 different flux density ranges of the $A 11-8 \mathrm{ky}$, the $3 C R$ and the Ooty occultation surveys. A more general function $\mathrm{N}(\mathrm{S}, \theta, \mathrm{z})$ or $\mathrm{N}(\mathrm{S}, \theta, \mathrm{m})$, where $\mathrm{z}$ is the redshift and $\mathrm{m}$ the apparent magnitude is a lso discussed briefly - this function broadly represents the map of the radio universe and combines several relations $N(S), N(\theta)$, $N(z), S(\theta), \quad(z), z(S)$ discussed by earlier a uthors. 


\section{OBSER VATIONS AND RESULTS}

Angular size statistics of 163 weak extragalactic radio sources observed at $327 \mathrm{MHz}$ by the method of lunar occultation at Ootacamund during 1970-71 were presented recently by Swarup (1975) who compared them with the angular extents of the stronger sources from the 3CR and the All-sky catalogues. The open triangles in Fig. 1 show the median values $\theta \mathrm{m}$ for an independent sample of 119 radio sources observed at Ootacamund during 1973-74. The new values show a good agreement with the values derived by Swarup (1975) from the earlier sample of 163 sources (closed triangle). It is also seen that median values based on the recent high resolution maps of $3 \mathrm{CR}$ sources by Pooley and Henbest (1974), Riley and Pooley (1975) and other recent Cambridge papers, as shown by crosses in Fig. 1, are almost the same as the values derived from the earlier data by Swarup (filled circles).

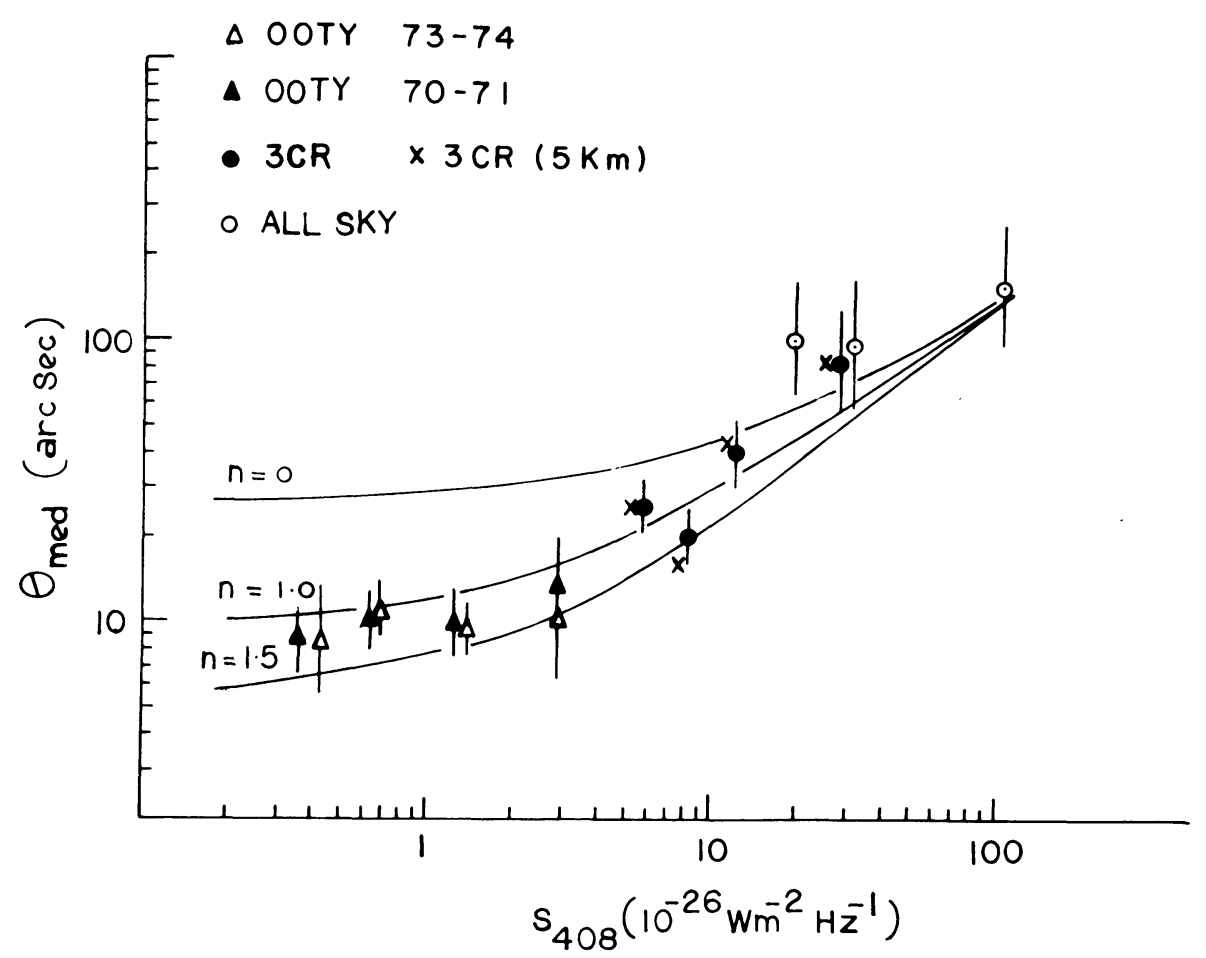

FIG. 1: The median values of angular size $\theta_{\mathrm{m}}$ for the new sample of 119 sources observed during 73-74 are compared with the earlier values found for 70-71 data. Also the $\theta_{\mathrm{m}}$ values for the $3 \mathrm{CR}$ sources found by Swarup (1975 - filled circles) have been revised to include recent high resolution observations. The vertical lines show statis tical errors. The 3 curves are theoretical predictions from Fig. 8 by Kapahi (1975). 


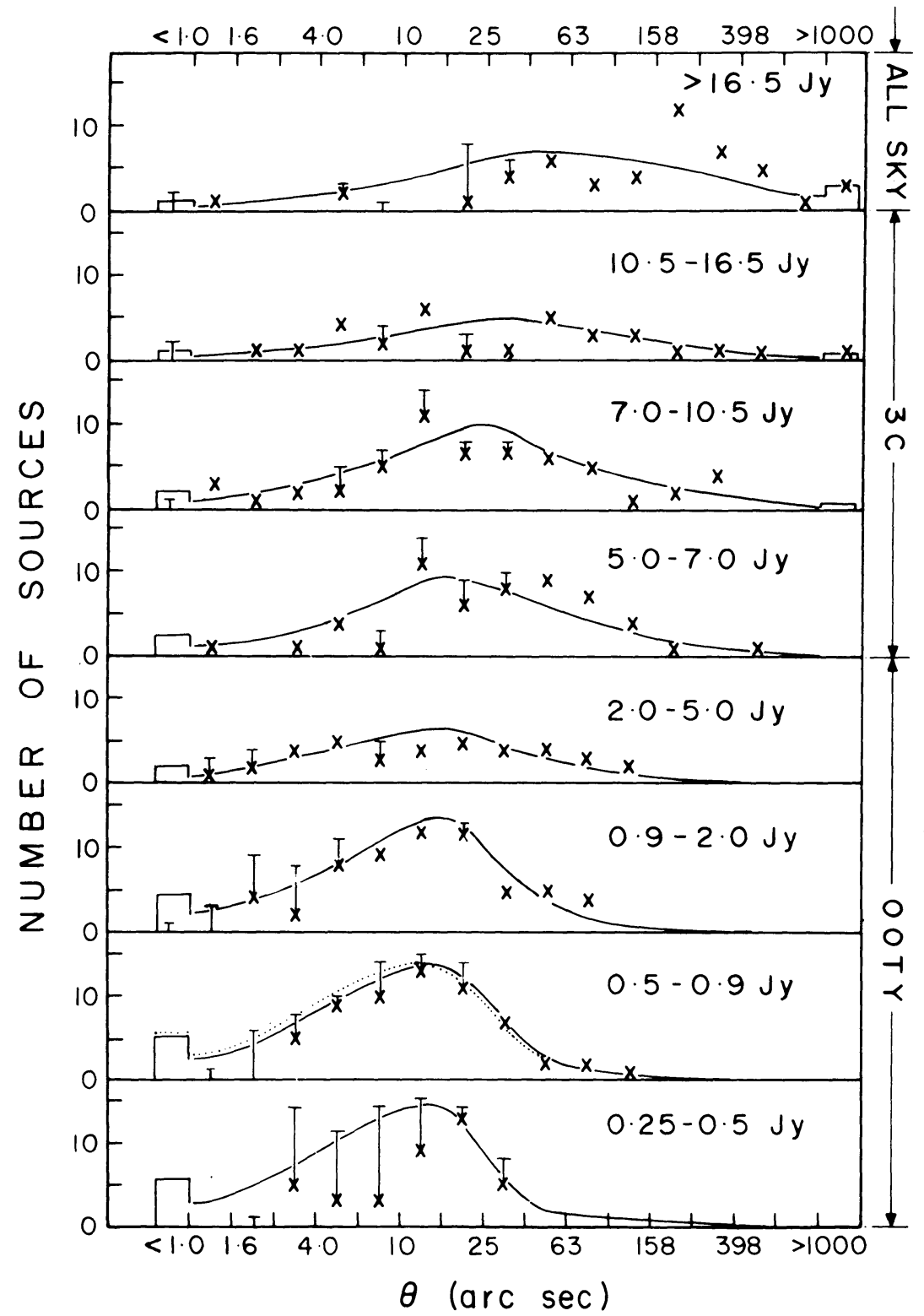

FIG. 2: The observed number of sources and theoretical predictions with angular size in the intervals shown at the top a nd bottom are plotted for 8 different flux density ranges of the All-sky, 3C and Ooty sources. The observed definite values are shown by crosses and upper limits by bars and theoretical predictions by full line curves. Some $3 \mathrm{C}$ sources have flux below $5 \mathrm{Jy}$ but a re included in the range 5 to $7 \mathrm{Jy}$ and a few Ooty sources with flux $5 \mathrm{Jy}$ are included in 2 to $5 \mathrm{Jy}$ range. 
In Fig. 2 are given distribution of angular sizes of 513 radio sources comprising of (a) 62 radio sources with $S_{408}>16.5 \mathrm{Jy}$ from the All-sky Catalogue (Robertson, 1973), (b) 197 sources of the complete sample from the $3 C R$ survey, including 28 sources with $\mathrm{S}_{408}>16.5 \mathrm{Jy}$ on Wyllie's scale which form part of the above All-sky sample and (c) 282 Ooty occultation sources observed during 1970-71 and 1973-74. The definite values of number of sources $\mathrm{N}$ are shown by crosses and upper limits by bars. The full line curves are theoretical predictions for the evolutionary model as defined in Table I of the paper by Kapahi (1975), except that we have taken the value of the exponent $n=1.1$ for the linear size evolution proportional to $(1+z)^{-n}$ because it provides a better fit to the data.

Since the area of the sky covered by Ooty Survey is not known accurately, we have normalized the total number of sources for the theoretical curve in each flux density range to be equal to the observed number. It may be noted anyway that Ka pahi's luminosity function (1975) gives a reasonable fit to the source counts, $N(S)$, observed by the Cambridge group. For a statistical comparison of the theoretical calculations (solid lines) and experimental data of Fig. 2 we have considered 72 bins with $\log \theta \leqslant 0.6,0.6$ to $0.8, \ldots, 1.8$ to 2.0 and $>2.0$ for each of the 8 flux density ranges. There are, however, only 64 degrees of freedom as we have normalized for the total number of sources in the 8 ranges as noted above. The standard chi-square test gives values of $74,71,73$ and 104 for $n=1.0,1.1$, 1.2 and 1.5 respectively, corresponding to a probability of about 30 percent for the first three and about 0.02 percent for the last. Kapahi's 3 -slope luminosity function (1976) also gives a chi-square of 71 for $n=1.1$ and density evolution parameter $\beta=6.0$.

It should be noted that the observed values of the maximum angular size $\theta$ for the Ooty sources are smaller than the true values because these sources have been scanned along two or three position angles only. To minimize these projection effects, only those sources have been included in the sample of 282 Ooty sources for which the difference between the position angles of the scans is between $30^{\circ}$ to $150^{\circ}$. Further, since the major axes of the radio sources are expected to be randomly distributed with respect to the scanning direction, it can be shown that the projection effects essentially shift the theoretical curves towards lower values of $\theta$ by a 8 mall a mount as shown by the dotted line for the range 0.5 to $0.9 \mathrm{Jy}$. With these corrections a value of $n=1.0$ is favoured.

The theoretical curves in Fig. 2 predict that there are less than $8 \%$ sources with $\theta>40$ arc sec around $1 \mathrm{Jy}$. In order to ver if 


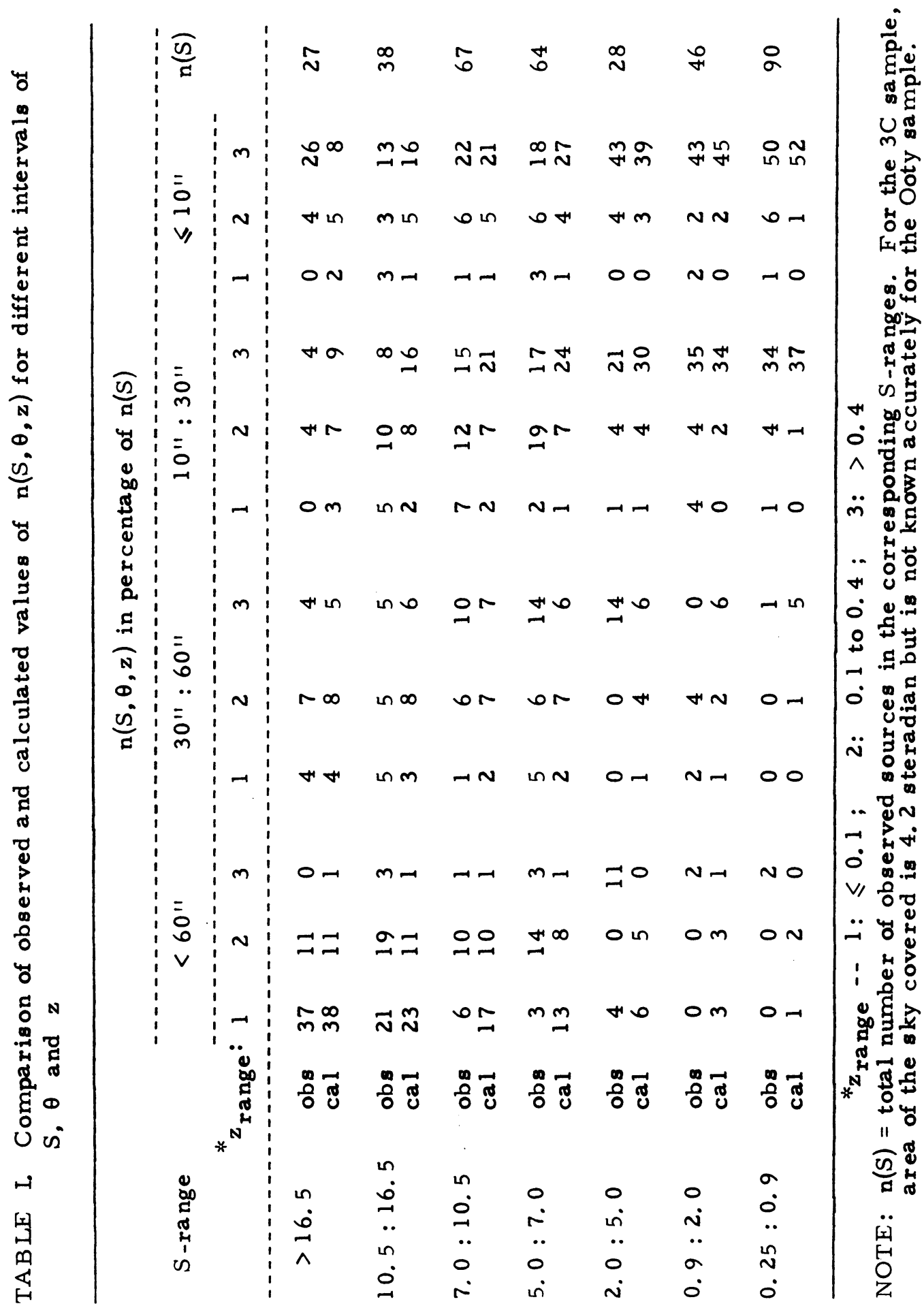


whether the occultation method has excluded detection of any large diameter sources of low flux densities, we have formed a sample of 43 sources from Molonglo and Bologna catalogues with $\mathrm{S}_{408}>0.5 \mathrm{Jy}$ (median value $\sim 1.2 \mathrm{Jy}$ ) and which were expected to be occulted by the moon during the period of observations at Ootacamund, but excluding sources with $|\mathrm{b}|<10^{\circ}$ and also if the difference of position angles of the observed strip scans by the occultation method was $<30^{\circ}$. Occultations were clearly seen for 41 sources and only 6 or 7 out of 43 sources have $\theta>40$ arc sec, which agrees reasonably with the expectation from Fig. 2.

\section{DISC USSIONS}

It is seen from Fig. 2 that the evolutionary model provides a good fit to the observed $N(S, \theta)$ distribution. The Steady State model has a poor fit with chi-square values corresponding to probabilities less than $10^{-6}$ and the $\mathrm{fit}$ is much poorer if the source counts are also considered.

In making chi-square test we have given equal weight to all the flux density ranges. In fact one should give equal weight to equal volumes of space. Also, other observable parameters such as spectral index distribution, redshift and/or apparent magnitude dis tribution need to be considered simultaneously. As shown in Table I, we have made a preliminary comparison of the theoretical predictions for a 3-slope luminosity function by Ka pahi (1976) with the experimental data of $\mathrm{N}(\mathrm{S}, \theta, z)$ and find a reasonably good agreement. Unidentified sources were assumed to have $z>0.4$ and for sources with no redshifts it was assumed that the absolute magnitude of the associated optical object was -23 for radio galaxies and -25.8 for QSOs. For the weaker sources, any spread in the optical luminosity function may be important.

To conclude, the flux density-angular size distribution provides independent evidence for the evolutionary model of the universe. With improved data it may be possible to derive the parameters of the model more accurately.

\section{ACKNOW LEDGEMENT}

One of us (G. S. ) was a recipient of a Jawaharlal Nehru Fellowship during this period. 


\section{REFERENCES}

Kapahi, V. K. 1975, Mon. Not. R. astr. Soc., 172, 513.

Ka pahi, V. K. 1976, Private Communication.

Pooley, G. G. , Henbest, S. N. 1974, Mon. Not. R. astr. Soc., 169, 477.

Riley, J. M. , Pooley, G. G. 1975, Memoirs R. astr. Soc. , 80, 105. Robertson, J. G. 1973, Austr. J. Phys., 26, 403.

Swarup, G. 1975, Mon. Not. R. astr. Soc., 172, 501.

\section{DISCUSSION}

P. Vêrron: You have used in your model a redshift cut-off of $z=3$ which may be a little unrealistic. What would happen if you were using instead $z=4$ or 5 ?

Kapahi: At the flux levels of the Ooty data the value of the cut-off redshift is not very critical.

Readhead: There are some difficulties associated with the detection of radio sources of angular size greater than a few hundred arc seconds by lunar occultations. Could you describe briefly how your analysis procedure takes account of these, and could you give us a figure for the limiting surface brightness detectable with the Ooty telescope? How many large sources (if any) might have been missed in the survey?

Swarup: The occultation observations are made using the "phase-switched" mode of the Ooty radio telescope, in which the two halves are multiplied together, so that the total drift due to movement of the moon in declination is less than $5^{\circ} \mathrm{K}(\sim 2 \mathrm{Jy})$ over a period of several hours. However, over $2 / 3 \mathrm{rd}$ of the moon's disc the drift is less than about $1^{\circ} \mathrm{K}$ over periods of few hours. Thus the number of sources of diameter more than about 100 arc second and flux $\approx 1$ Jy which have been missed are likely to be insignificant, particularly because the measured distributions cut off at a much lower value of the angular size.

Conway: Is it possible that the "angular size" quoted is a mixture in some cases of "Largest Angular Size", i.e. isophotal diameter, and in other cases of Component Separation. Would it be possible to restrict the data, so as to be homogeneous, only to include Component Separations?

Swarup: The "largest angular size" $\theta$ defined by us refers not to the separation of the outermost components for the double or complex sources but to the half-power width for unresolved or single sources (see Swarup, MNRAS, 172, 501, 1975). But since more than $80 \%$ of the sources are found to be double, the effect of the mixture is considered small. In any case, with the availability of the detailed maps made with the $5 \mathrm{~km}$ telescope for the $3 \mathrm{CR}$ complete sample and higher resolution observations at Ooty, it is planned to investigate finer features of $N(s, \theta)$ distribution. 
A STATISTICAL ANALYSIS OF THE ANGULAR SIZE FLUX DENSITY RELATION

J.V. Narlikar

This is a brief description of the investigation by Dr. S.M. Chitre and myself, of the $\theta-s$ relation from the ooty and 3CR surveys. The $\theta$-s diagram has considerable scatter of the wide range of power and size distributions of radio sources, as well as the projection effects. Indeed, the scatter in the $\theta-s$ is worse than that in the optical m-z relation, even for the QSO's. Just as it is impossible to determine the Hubble constant reliably from the $\mathrm{m}-\mathrm{z}$ plot for the QSO's alone, so it appears ambitious to arrive at unambiguous cosmological conclusions from the present $\theta-s$ data. A single statistic like $\theta_{m}(s)$ used by Drs Swarup and Kapahi cannot do justice to the information content of the $\theta-s$ scatter diagram.

To show this we have used two independent tests of the data. The first one is a modified form of the familiar $\chi^{2}$-test which takes into account the variable (and unknown) areas of the sky covered by the Ooty survey. The second test uses ranking techniques which properly take into account the dispersion of the median $\theta_{m}(s)$. The tests are used to compare the observed plot with that predicted by evolutionary and non-evolutionary models. A wide range of models of both types are consistent with the data at 18 level. The Kapahi model for $\mathrm{n}=1.5$ appears to be ruled out by the $\chi^{2}$-test while a non-evolutionary model with a mild power-size correlation survives. Data with considerably reduced scatter is needed to draw any meaningful cosmological conclusion.

Kapahi: It seems to me that in comparing the predictions of the Steady State and the evolutionary model with the observed $\theta-s$ correlation, Dr. Narlikar has chosen rather unrealistic parameters for the luminosity function and the size function which minimize the discrepancy between the steady state and the observations. For the evolutionary model however, he has used a value of $n=1.5$ which is not the best fit value.

Narlikar: In choosing the parameters described by me other checks besides the $\theta-s$ relation were performed. For example, the $N-\theta$ relation was taken into account in the $3 C-R$ part in the same way as done by you. Also, a predicted nearby sample on the basis of this model does not seem to disagree with what we know about the luminosity-size distribution of radio sources.

As regards the second point, the cases $n=1,1.1$ etc. have not been examined in this way. Prof. Swarup tells me that he finds the minimum $\chi^{2}$ for these cases is better than for $n=1.5$. In that case, it illustrates the power of the proposed minimum $\chi^{2}$ test in distinguishing between different values of $\mathrm{n}$. My own interest in the problem was not to look for the best $n$ but to show that at present the data does not rule out non-evolutionary models. 